\title{
Correction to: Personalized Multimorbidity Management for Patients with Type 2 Diabetes Using Reinforcement Learning of Electronic Health Records
}

\author{
Hua Zheng ${ }^{1} \cdot$ llya O. Ryzhov ${ }^{2} \cdot$ Wei Xie $^{1} \cdot$ Judy Zhong ${ }^{3}$ (D)
}

Published online: 25 February 2021

(c) Springer Nature Switzerland AG 2021

\section{Correction to: Drugs \\ https://doi.org/10.1007/s40265-020-01435-4}

Corresponding author, which currently reads

Correspondence to Judy Zhong

Should read

Correspondence to either Wei Xie or Judy Zhong

The original article has been updated.

The original article can be found online at https://doi.org/10.1007/ s40265-020-01435-4.

Wei Xie

w.xie@northeastern.edu

$\triangle$ Judy Zhong

judy.zhong@nyulangone.org

1 Department of Mechanical and Industrial Engineering, Northeastern University, 360 Huntington Avenue, Boston, MA, USA

2 Robert H. Smith School of Business, University of Maryland, College Park, MD, USA

3 Division of Biostatistics, Department of Population Health, New York University School of Medicine, NYU Langone Health, 180 Madison Avenue, 4th Floor, Room 452, New York, NY 10016, USA 